\title{
CHEMICAL MODIFICATION OF TANNIN/FURANIC RIGID FOAMS BY ISOCYANATES AND POLYURETHANES
}

\author{
X. $\mathrm{Li}^{1}$,2, M.C. Basso ${ }^{2}$, V. Fierro ${ }^{2}$, A. Pizzi ${ }^{1 \wedge}$, A. Celzard ${ }^{2}$
}

\begin{abstract}
Tannin/furanic rigid foams were modified by either polymeric 4,4' diphenylmethane diisocyanate (pMDI) or a one-component polyurethane still presenting reactive isocyanate groups (PUR). Several different sequences of mixing the isocyanate and polyurethane with the chemicals used to prepare tannin-based foams were tested. Scanning electron microscopy (SEM) showed that addition of either pMDI or PUR yielded a more ordered microstructure of the foam and a larger cell size. Series of two different cell sizes were noticed: those obtained by the evaporation of the blowing agent used for classical tannin-based foams and those caused by the formation of $\mathrm{CO}_{2}$ issued by the reaction of the isocyanate groups with water. The cell size, density, thermal conductivity and mechanical property of tannin/furanic foams were controlled by changing the amount of blowing agent and weight fraction of pMDI. The cell size of pMDI modified foams was largest when the weight fraction of pMDI was $5 \%$ and kept decreasing as the pMDI percentage became higher. The density of the foams increased with the increase of the weight fraction of pMDI. The addition of pMDI increased slightly the thermal conductivity of tannin/furanic foam with the foams still presenting good thermal insulation. Moreover, the addition of pMDI in tannin/furanic foams improved markedly the foam's mechanical properties.
\end{abstract}

Keywords: Tannin/furanic rigid foams, Isocyanate modification, Polyurethane modificaton, Microstructure, Mechanical properties.

\section{INTRODUCTION}

Tannin/furanic rigid foams are polymeric materials used in the field of packaging, crash protection, catalyst supports and metal ion adsorption (Meikleham and Pizzi 1994, Pizzi et al. 2008, Tondi and Pizzi 2009, Tondi et al. 2009a,b,c,d). Because of their valuable characteristics of good fire resistance and low thermal conductivity, Tannin/furanic rigid foams are particularly useful as insulating materials (Tondi et al. 2008, 2009a). Tannin/furanic rigid foams are networked structures obtained from a highly viscous liquid phase composed of a tannin-formaldehyde resin mixed with furfuryl alcohol (FA). FA is used both for the heat generation due to the exothermic self-condensation and for its polycondensation with the tannin and formaldehyde under acid conditions (Meikleham and Pizzi 1994, Choura et al 1996). After adding the acid reaction catalyst the foaming process starts and is completed within 2-5 minutes. The expansion to a low density foam of the viscous liquid is caused by the evaporation of a low temperature boiling solvent during the exothermic reaction. Controlled pyrolysis of such materials yields a reticulated vitreous carbon (RVC) having new properties, such as electrical conductivity, higher mechanical strength and even improved resistance to very high temperatures and chemicals (Tondi et al. 2009c,d). Thus, the applications may be extended to porous electrodes, catalytic filters or in carbon composites.

\footnotetext{
${ }^{1}$ ENSTIB-LERMAB, Nancy-Université, 27 rue Philippe Séguin, BP1041, 88051 Epinal cedex 9, France.

${ }^{2}$ Institut Jean Lamour - UMR CNRS 7198, CNRS - Nancy-Université - UPV-Metz, Département Chimie et Physique des Solides et des Surfaces. ENSTIB, 27 rue Philippe Séguin, BP 1041, 88051 Epinal cedex 9, France.

Corresponding author: ^antonio.pizzi@enstib.uhp-nancy.fr

Received: 11.11. 2011 Accepted: 05.04. 2012.
} 
Polymeric 4,4' diphenylmethane disocyanate (pMDI) and one-component polyurethane still presenting reactive isocyanate groups (PUR) could react during foaming process of tannin/furanic system (Frisch et al. 1983), thus could modify the skeleton and microstructure of tannin/furanic rigid foams. For the foamed materials, not only the nature of the skeleton material properties, but also the microstructure of the foam plays a very important role on the properties of the foams, such as insulation, compressive strength and Young's modulus. So in this paper, pMDI and PUR were added in order to improve these properties of our foams. However, the addition of pMDI and PUR could highly improve the viscosity of the system. Especially when pMDI is added, the mixture acquires an almost gel-like state, which makes rather difficult to mix the foam chemical components. In order to get foams more homogeneous, of lower thermal conductivity and higher compressive strength, five different mixing sequences were tried.

\section{EXPERIMENTAL}

\section{Materials}

Commercial tannin, obtained by spray drying of aqueous Mimosa (Acacia mearnsii formerly mollissima, De Wildt) bark extracts and containing 84\% phenolic material (Silva Chimica, St.Michele Mondovi, Italy), was used to synthesize the foams. The single component polyurethane (PUR) used was Mirapur 9521 (Geitslich, Schlieren, Switzerland) and the polymeric pMDI of viscosity 199 $\mathrm{mPa}$ 's at $25^{\circ} \mathrm{C}$ was from DOW Chemicals. The formulation used is shown in table 1. para-Toluene sulphonic acid (pTSA) was added as a $65 \%$ water solution and the formaldehyde we used is $37 \%$ water solution. Diethyl ether (DE) was used as the blowing agent

Table 1. Formulation of pMDI modified tannin/furanic rigid foams.

Formulation used for $\mathrm{p} 1$ to $\mathrm{p} 7$ included.

\begin{tabular}{cccccccc}
\hline Component & Tannin & pTSA & FA & Water & Formaldehyde & DE & PUR/pMDI \\
Amount $(\mathrm{g})$ & 30 & 11 & 10.5 & 6 & 7.4 & 3 & $8.5(10 \%)$ \\
\hline
\end{tabular}

\section{Preparation of the foams}

Different mixing sequences of the materials were tried to optimize the results obtained. The ones that gave best results were as follows:

Mixing sequence 1. Tannin and pMDI were first mixed together. After stirring by a general blender, the mixture of FA, formaldehyde, water and DE were added. The whole mixture was stirred strongly by the blender and after $20 \mathrm{~s}$ pTSA was added followed by $20 \mathrm{~s}$ strong stirring by the blender.

Mixing sequence 2. FA, formaldehyde, water DE, and pMDI were first mixed together. After stirring by the blender, the mixture was added to tannin. The whole mixture was stirred strongly by the blender and after $20 \mathrm{~s}$ pTSA was added followed by $20 \mathrm{~s}$ strong stirring by the blender.

Mixing sequence 3 . Tannin and pMDI were first mixed together, and then FA was added. After strong stirring by the blender, the mixture of formaldehyde, water and DE were added. The whole mixture was stirred strongly by the blender and after $20 \mathrm{~s}$ pTSA was added followed by $20 \mathrm{~s}$ strong stirring by the blender.

Mixing sequence 4. The mixture of FA and water was added into the mixture of tannin and pMDI. After strong stirring by the blender the mixture of formaldehyde and DE was added. The whole 
mixture was stirred strongly by the blender and after 20 s pTSA was added followed by 20 s strong stirring by the blender.

Mixing sequence 5. The mixture of FA, formaldehyde, water, and DE was added into a $250 \mathrm{~mL}$ beaker already containing the tannin, and the whole stirred strongly by the blender for $10 \mathrm{~s}$. The pMDI was then added to the viscous mixture. The whole mixture was stirred strongly by the blender and after $20 \mathrm{~s}$ pTSA was added followed by $20 \mathrm{~s}$ strong stirring by the blender.

A black foam was obtained within 2 minutes after the adding of pTSA. The five mixing sequences were prepared holding the PUR constant at $10 \mathrm{wt} \%$. For pMDI, preparation followed the procedures of mixing sequences 4 and 5 and the corresponding samples were called p6 and p7. All the foams were prepared according to Table 1, and left to harden and age for 3 days. For comparison, the standard tannin/furanic foam, p0, was prepared according to table 1 only without pMDI.

Based on mixing sequence 5 and formulation table 1, pMDI was maintained in a fixed proportion of $10 \%$ and the blowing agent $\mathrm{DE}$ was varied between $1 \mathrm{~g}$ and $5 \mathrm{~g}$, and the samples obtained were named DE-1, DE-2, DE-4 and DE-5, correspondingly. Afterwards the weight fraction of pMDI were changed to $5 \%, 15 \%$ and $20 \%$ while maintaining the same proportions of all the other components in table 1 , and the samples obtained were designated $\mathrm{p}-5 \%, \mathrm{p}-15 \%$ and $\mathrm{p}-20 \%$.

\section{Measurements}

\section{Bulk and skeletal density}

Blocks of foam of dimension of $3 \times 3 \times 1.5 \mathrm{~cm}^{3}$ were weighted to obtain bulk density. Skeletal density, sometimes called true density, was evaluated by helium pycnometry in a lab-made apparatus. The cellular morphologies of the foams were obtained by scanning electron microscope (SEM Hitachi S 4800). Thermal conductivity of foam samples of dimension of $3 \times 3 \times 1.5 \mathrm{~cm}$ was measured by the transient plane source method (Hot Disk TPS 2500) at room temperature. The mechanical resistance to compression was investigated with an Instron 4206 universal testing machine at a load rate of 2.0 $\mathrm{mm} \mathrm{min}^{-1}$.

\section{RESULTS AND DISCUSSION}

\section{Density and Porosity}

After preparation, in $\mathrm{p} 1$ and $\mathrm{p} 2$ some small white plastic-like particles were observed. This is consequence of the agglomeration and poor dispersion of the pMDI. Thus p1 and 2 gave heterogeneous materials, badly mixed and of unmanageable viscosity. As a consequence these two procedures are not discussed further. The bulk density $d$ and skeletal density $d_{s}$ for p3, p4, p5, p6 and p7 are shown in table 2 . The porosity $P$ of the samples were calculated by (Tondi et al. 2009a)

$$
P=1-d / d_{s}
$$

where $d$ is the bulk density and $d_{s}$ is the skeletal density. The porosity of all the samples is in the range of 91 94\%. For the foams modified with the PUR there was not much difference of apparent density and porosity. However, for the pMDI modified foams p6 showed a lower density and a higher porosity. 
Table 2. Density, porosity and thermal conductivity of pMDI modified tannin/furanic foams.

\begin{tabular}{ccccc}
\hline & $\begin{array}{c}\text { Skeletal density } \\
\mathrm{g} / \mathrm{cm}^{3}\end{array}$ & $\begin{array}{c}\text { Apparenty density } \\
\mathrm{g} / \mathrm{cm}^{3}\end{array}$ & $\begin{array}{c}\text { Porosity } \\
\%\end{array}$ & $\begin{array}{c}\text { Thermal conductivity } \\
\mathrm{W} / \mathrm{mK}\end{array}$ \\
\hline p0 & $1.310 \pm 0.005$ & 0.065 & 96 & $0.044 \pm 0.002$ \\
p3 & $0.132 \pm 0.003$ & 1.36 & 91 & $0.055 \pm 0.002$ \\
p4 & $0.119 \pm 0.005$ & 1.4 & 92 & $0.054 \pm 0.002$ \\
p5 & $0.116 \pm 0.002$ & 1.41 & 92 & $0.054 \pm 0.001$ \\
p6 & $0.082 \pm 0.002$ & 1.3 & 94 & $0.047 \pm 0.001$ \\
p7 & $0.103 \pm 0.004$ & 1.44 & 93 & $0.054 \pm 0.001$ \\
\hline
\end{tabular}

The relationship between the percentage of pMDI and bulk density and porosity is shown in table 3. The bulk density increased and the porosity decreased when the proportion of pMDI increased. The data fit the following linear regression relationships

$$
\begin{gathered}
P=0.96-0.34 * f \quad\left(\mathrm{R}^{2}=0.990\right) \\
d=0.0554+0.562 * f \quad\left(\mathrm{R}^{2}=0.930\right)
\end{gathered}
$$

where $f$ is the weight fraction of pMDI and $d$ is the bulk density. With $10 \%$ of pMDI, when varying the amount of DE, the bulk density and porosity are shown in table 5 . The bulk density decreased rapidly and porosity increased with the increase in the amount of DE. Thus, in pMDI modified tannin foams the density can be modified by changing the amount of $\mathrm{DE}$.

Table 3. Physical properties of pMDI modified tannin/furanic foams.

\begin{tabular}{cccccc}
\hline & $\begin{array}{c}\text { Bulk density } \\
\text { g/cm }\end{array}$ & $\begin{array}{c}\text { Porosity } \\
\%\end{array}$ & $\begin{array}{c}\text { Thermal } \\
\text { conductivity } \\
\text { W/mK }\end{array}$ & $\begin{array}{c}\text { Compressive } \\
\text { strenghth } \\
\mathrm{MPa}\end{array}$ & $\begin{array}{c}\text { Young`s modulus } \\
\mathrm{MPa}\end{array}$ \\
\hline DE-1 & 0.274 & 81 & $0.065 \pm 0.002$ & $5.22 \pm 0.05$ & $148.50 \pm 5.3$ \\
DE-2 & 0.166 & 88 & $0.056 \pm 0.001$ & $2.55 \pm 0.05$ & $54.30 \pm 3.2$ \\
p7 & 0.103 & 93 & $0.054 \pm 0.001$ & $0.47 \pm 0.02$ & $3.37 \pm 0.52$ \\
DE-4 & 0.076 & 95 & $0.049 \pm 0.001$ & $0.20 \pm 0.01$ & $0.65 \pm 0.01$ \\
DE-5 & 0.070 & 95 & $0.055 \pm 0.002$ & $0.16 \pm 0.02$ & $0.64 \pm 0.02$ \\
p0 & 0.065 & 96 & $0.044 \pm 0.001$ & $0.15 \pm 0.01$ & $3.60 \pm 0.10$ \\
p-5\% & 0.081 & 94 & $0.049 \pm 0.001$ & $0.36 \pm 0.02$ & $3.24 \pm 0.12$ \\
p-15\% & 0.126 & 91 & $0.052 \pm 0.002$ & $1.73 \pm 0.04$ & $7.27 \pm 0.31$ \\
p-20\% & 0.183 & 89 & $0.056 \pm 0.002$ & $2.57 \pm 0.04$ & $62.80 \pm 0.6$ \\
\hline
\end{tabular}

Note: DE-3 is the same sample with $\mathrm{p} 7$

\section{Microstructure of the foams}

Examples of the cellular structures of the samples are shown in figure 1. For the SEM image of p0, the cells were small, broken and disordered. However, for the foams with pMDI, each cell is connected with its neighbours through more or less circular windows. Moreover, the cell size of the foams with pMDI was larger than p0. It is very interesting to notice that there are many small cells 
among the big cells, thus that two different cell sizes are obtained when pMDI is present. The smaller cells are produced by a different mechanism with the blowing of $\mathrm{DE}$, namely the production of $\mathrm{CO}_{2}$ resulting by the reaction of the $-\mathrm{N}=\mathrm{C}=\mathrm{O}$ groups of $\mathrm{pMDI}$ with water (Frisch et al. 1983).
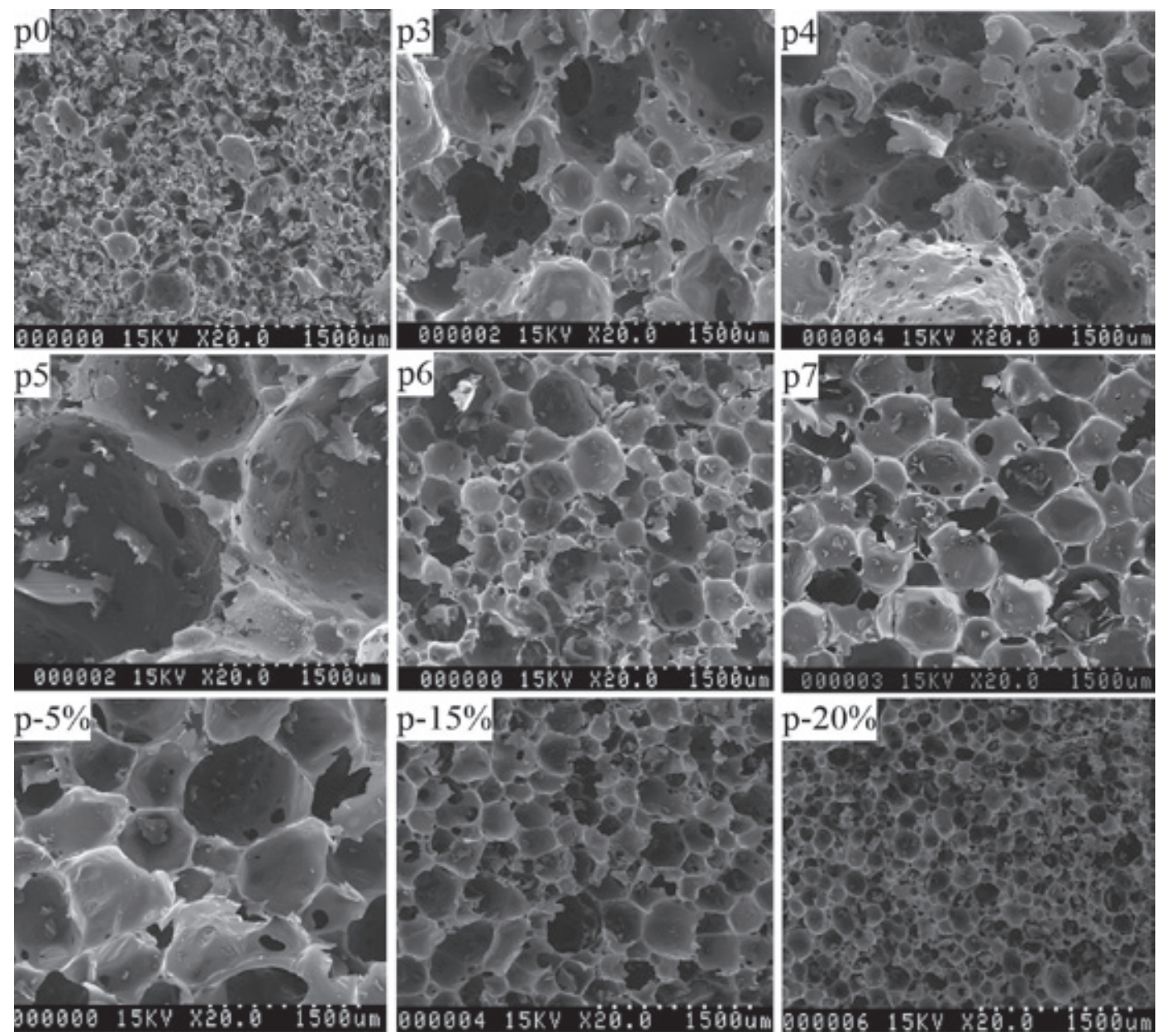

Figure 1. SEM micrographs of pMDI modified tannin/ furanic rigid foams. p0, p3, p4, p5, p6, p7 mixing sequences (see text). p-5\%, p-15\%, p-20\% difference percentages of pMDI.

\section{Thermal conductivity}

Thermal conductivities of the samples are presented in table 3. The Thermal conductivities of pMDI modified tannin/furanic foams are about $0.054 \mathrm{~W} / \mathrm{m} \cdot \mathrm{K}$ thus slightly higher than the foams without pMDI (at $0.44 \mathrm{~W} / \mathrm{m} \cdot \mathrm{K}$ ). This may be caused by the decrease of porosity after the addition of the pMDI. The foam $\mathrm{p} 6$, which had the highest porosity, had the lowest thermal conductivity at $0.47 \mathrm{~W} / \mathrm{m} \cdot \mathrm{K}$. Thus, the results do not show too much difference in thermal conductivity for the foams with and without $\mathrm{pMDI}$, at least no statistically significant difference ( $\mathrm{P}$ value $=0.325$ at the 0.05 level). The thermal conductivity of the foams shown in Table 3 indicates that when the weight fraction of pMDI was under 20\%, the thermal conductivity increased gradually. Fitting the results in Table 3 according to a linear regression shows,

$$
k=0.0456+0.054 * f \quad\left(\mathrm{R}^{2}=0.828\right)
$$


where $k(\mathrm{~W} / \mathrm{m} \cdot \mathrm{K})$ is thermal conductivity, $f$ is the weight fraction of pMDI. Eq. 4 shows that $k$ increases very slowly as $f$ increases. The results do not show much difference in thermal conductivity for the foams with and without pMDI

\section{Compressive strength}

For tannin/furanic foams, the stress-strain curves always show three distinct regions: linear elastic, collapse and densification. These three regions are also shown in PUR and pMDI modified carbon/furanic foams (Figure 2). PUR modification p3 had a better compression resistance than p4 and $\mathrm{p} 5$, and better than the pMDI modified foams $\mathrm{p} 6$ and $\mathrm{p} 7$, where $\mathrm{p} 6$ had a better compression resistance than p7. Both $\mathrm{p} 3$ and p6 were mixed with mixing sequence 3 (cf. Experimental).
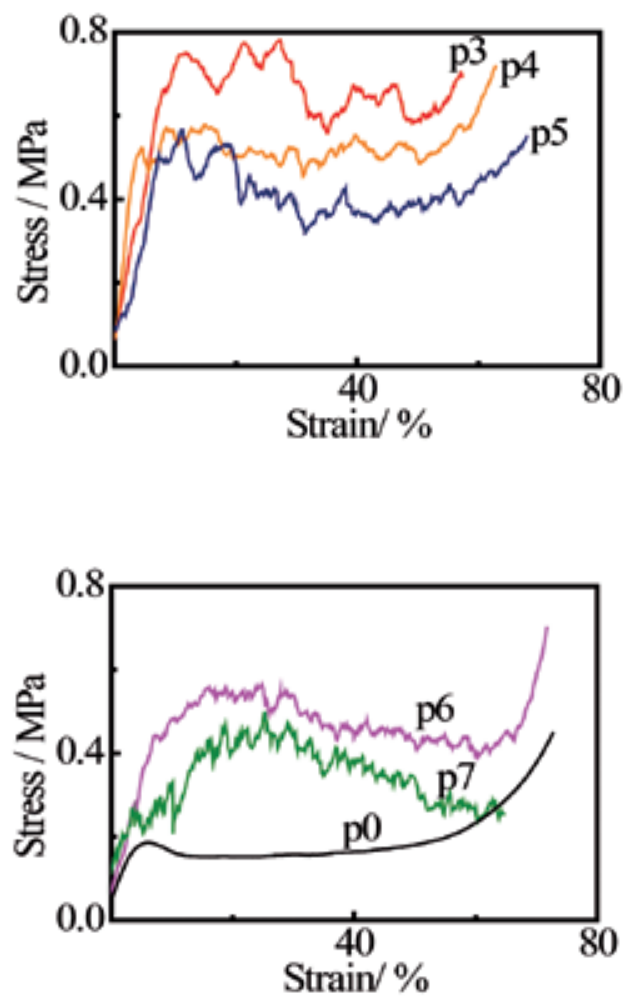

Figure 2. Stress-strain curves of pMDI modified tannin/furanic foams.

a) With different percentage of pMDI; b) With different amount of DE.

The stress-strain curves of the pMDI modified tannin/furanic foams (Figure 3). The foams behaved as typical elastic fragile cellular solids. Moreover, as the percentage of pMDI increased, the compressive strength improved. As the Diethyl ether decreased, hence the density increased, the compressive strength increased too. Especially when the amount of diethyl ether was lower than $3 \mathrm{~g}$, the compressive strength increased rapidly. The compressive strength and the Young`s modulus are shown in table 2 and figure 3. 


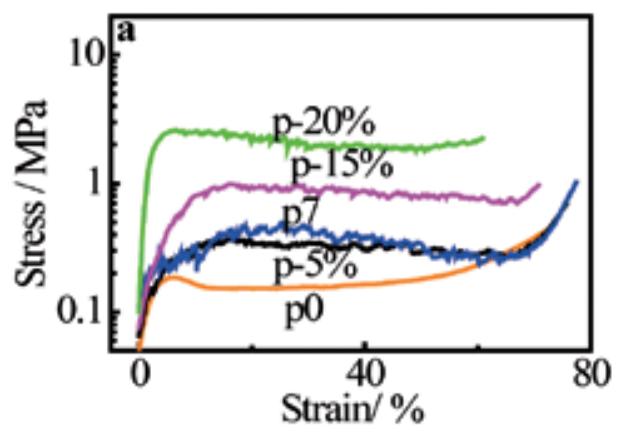

(a)

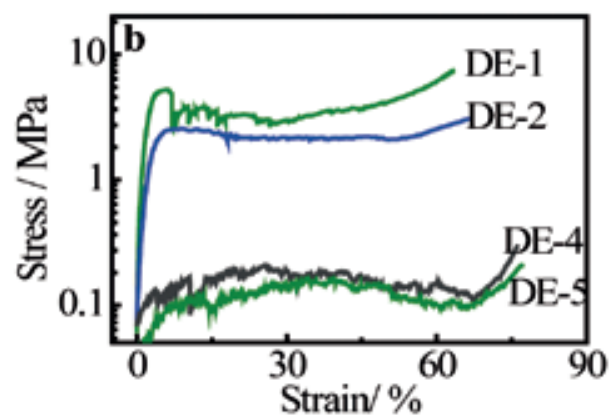

(b)

Figure 3. Stress-strain curves of pMDI modified tannin/furanic foams. a) With different percentage of pMDI; b) With different amount of DE.

The results in table 3 show that the compressive strength and the modulus of the foams can become as high as $5.22 \mathrm{MPa}$ and $148.5 \mathrm{MPa}$ respectively. Additionally, the compressive strength depends on the bulk density according to a power law (Sanders and Gibson 2003),

$$
\sigma=\sigma_{\mathrm{s}} * d^{n}
$$

where $\sigma_{s}$ is the yield strength of the (non-porous) solid and the exponent $n$ is associated with the structure and deformation mechanics of the cellular material. Theoretical values of $n$ are 1.5, 2 or higher and 1.36 if the foams are open-cells, closed-cells and hollow spheres. Fitting the results of table 2 to Eq.5, the following Eq. 6 was obtained,

$$
\sigma=212 * d^{2.69}
$$

It must be made clear that under the experimental conditions used Eq. 6 is only valid when the percentage of pMDI is $0 \%-20 \%$ range. The fitting result is shown in figure $4 . n=2.69$, and do not follow the theoretical values. This is considered as two different cell sizes caused by two different forming processes, as disused previously. Table 2 shows the compression strength and Young's modulus of all the pMDI modified tannin/furanic foams were much improved compared with p0. 


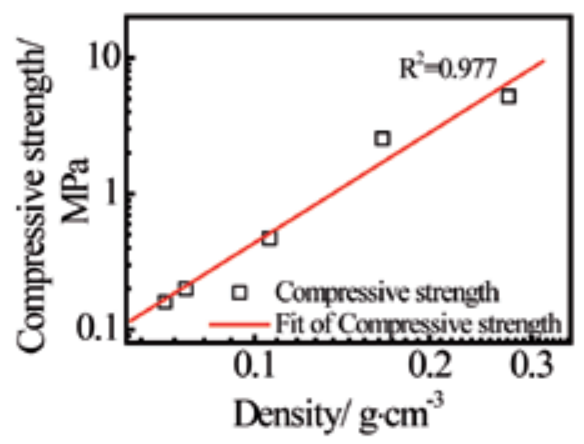

Figure 4. Relationship between bulk density of pMDI-modified tannin/furanic foams and their compression strength.

The results of the PUR modified foams are very similar to those of the pMDI modified foams, so we only report here the results for pMDI modified foams. The similarity of results was due to the PUR used containing a number of active isocyanate groups, thus there was no difference (other than for viscosity) in adding the PUR or the pMDI.

\section{CONCLUSIONS}

Two different kinds of fortifiers of tannin/furanic foams were used, namely a single component polyurethane (PUR) and polymeric 4,4' diphenylmethane diisocyanate. (pMDI). In the case of the PUR, better dispersion was achieved by mixing the furfuryl alcohol or the mixture of furfuryl alcohol and water into the mixture of tannin and PUR. For pMDI, it was better to add first the furfuryl alcohol into the mixture of tannin and pMDI. The density and cell size of the foams are larger for pMDI fortified foams and presents two sizes of cells, the smaller one being caused by the $\mathrm{CO}_{2}$ generated by the reaction of pMDI with water. The cell size of pMDI modified foams had a peak when the weight fraction of pMDI is $5 \%$. This opens a new window to control the sell size, which may play a very important role when our foam is used as catalyst carrier. The density of the foams increased with the increase of the weight fraction of pMDI. However, the density could be adjusted by varying the amount of blowing agent. The addition of pMDI enhanced the thermal conductivity of the foam but not drastically, the pMDI modified tannin/furanic foams being still good thermal insulators. Moreover, the addition of $\mathrm{pMDI}$ in tannin/furanic foams improved the mechanical property sharply, the compression strength was improved considerably for both PUR modified foams and pMDI modified foams, the greater the improvement the greater is the proportion of pMDI added. So the pMDI/PUR modified foams, with higher mechanical property and low thermal insulation, are supposed to be more suitable to use as thermal insulation layer of the house wall.

\section{ACKNOWLEDGEMENTS}

The authors gratefully acknowledge the financial support of the CPER 2007-2013 "Structuration du Pôle de Compétitivité Fibres Grand'Est” (Competitiveness Fibre Cluster), through local (Conseil Général des Vosges), regional (Région Lorraine), national (DRRT and FNADT) and European (FEDER) funds. The Chinese author gratefully acknowledges the bursary support of the China Scholarship Council (CSC). 


\section{REFERENCES}

Choura, M.; Belgacem, N.M.; Gandini, A. 1996. Acid-catalysed polycondensation of furfuryl alcohol: mechanism of chromophore formation and cross-linking. Macromolecules 29:3839-3842.

Frisch, K.C.; Rumao, L.P.; Pizzi, A. 1983. Diisocyanate wood adhesives in Wood Adhesives: Chemistry and Technology. Pizzi, A., Ed.; Marcel Dekker: New York, 1983; Vol. 1.

Meikleham, N.E.; Pizzi, A. 1994. Acid and alkali-setting tannin-based rigid foams. J. Appl. Polym. Sci. 53:1547-1556.

Pizzi, A.; Tondi, G.; Pasch, H.; Celzard, A. 2008. MALDI-TOF structure determination of complex thermoset networks: polyflavonoid tannin-furanic rigid foams. J. Appl. Polym. Sci. 110:14511456.

Sanders, W.S.; Gibson, L.J. 2003. Mechanics of hollow sphere foams. Mater. Sci. Eng. A 347: 70-85.

Tondi, G.; Pizzi, A. 2009. Tannin based rigid foams: characterisation and modification. Ind. Crops Prod. 29:356-363.

Tondi, G.; Zhao, W.; Pizzi, A.; Du, G.; Fierro, V.; Celzard, A. 2009a. Tannin-based rigid foams: a survey of chemical and physical properties. Biores. Technol. 100: 5162-5169.

Tondi, G.; Oo, C.W.; Pizzi, A.; Trosa, A.; Thevenon, M.F. 2009b. Metal adsorption of tanninbased rigid foams. Ind. Crops Prod. 29:336-346.

Tondi, G.; Fierro, V.; Pizzi, A.; Celzard, A. 2009c. Tannin-based carbon foams. Carbon 47 (6):1480-1492.

Tondi, G.; Pizzi, A.; Olives, R. 2008. Natural tannin-based rigid foams as insulation in wood construction. Maderas. Ciencia y Tecnología 10 (3):219-227.

Tondi, G.; Fierro, V.; Pizzi, A.; Celzard, A. 2009d. Erratum to tannin-based carbon foams. Carbon 47 (11):2761 p. 
